\title{
Recyclization of 5-hydroxy-2-pyrazolines in the reaction with N-nucleophiles
}

\author{
N. M. Kuz'menok, ${ }^{a}$ T. A. Koval'chuk, ${ }^{* a}$ and A. M. Zvonok ${ }^{b}$ \\ ${ }^{a}$ Department of Organic Chemistry, Belarusian State Technological University, 13A, Sverdlova str., \\ Minsk, 220050, Belarus \\ ${ }^{b}$ Center for Drug Discovery, Northeastern University, 360 Huntington Ave, Boston, MA 02115 USA \\ E-mail:kovtatale@yahoo.com
}

\section{Dedicated to Prof. Oleg Kulinkovich on the occasion of his 60th birthday}

\begin{abstract}
The reactions of 5-hydroxy-1-tosyl-2-pyrazolines with amines or with hydrazine have been investigated. We have shown that these compounds are convenient substrates for the synthesis of 5-aminosubstituted pyrazolines and NH-pyrazoles.
\end{abstract}

Keywords: Pyrazolines, pyrazoles, a ring-chain tautomerism

\section{Introduction}

Tautomeric transformations provide a valuable opportunity for the targeted synthesis of certain classes of compounds. ${ }^{1,2}$ The ring-chain transformations in the series of nitrogen-containing heterocycles allow rearranging the five-membered rings to the six-membered rings or reverse. In addition, various heteroatoms may be incorporated into the heterocyclic ring. ${ }^{2}$

It is known, that 5-hydroxy-2-pyrazolines in the solutions can exist in equilibrium with acyclic tautomers (Figure 1). A ratio of both forms is determined by the following factors: solvent, the structure of 1,3-dicarbonyl component and the nature of the substituted hydrazine moiety. ${ }^{3-7}$ Electron-withdrawing substituents in the $\beta$-dicarbonyl fragment favor the ring and the enhydrazine form, while electron-accepting groups at the nitrogen atom promote hydrazones and cyclic forms. In basic dipolar solvents the tautomeric equilibrium shifts towards the open forms, in non-polar solvents 5-hydroxy-2-pyrazoline structure is preferred.

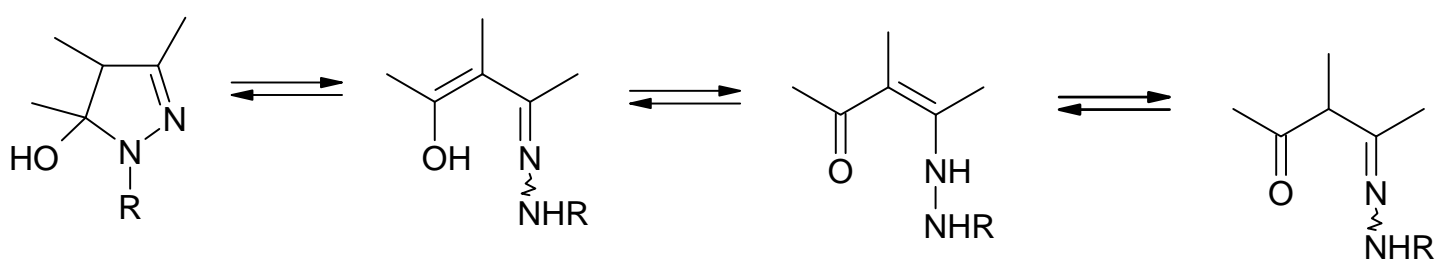

\section{Figure 1}


Previously, we synthesized several 5-hydroxy-1-tosyl-2-pyrazolines starting from unsaturated oxiranylketones. The mechanism of this reaction includes intra-molecular cyclization of the 1,3diketone tosylhydrazone intermediates. ${ }^{8}$ The presence of the reactive semi-aminal hydroxyl group $^{9,10}$ in 5-hydroxy-1-tosyl-2-pyrazolines allows their conversion to corresponding 5aminosubstituted pyrazoline derivatives upon the reaction with primary or secondary amines.

\section{Results and Discussion}

The series of 5-hydroxy-5-phenyl-1-tosyl-4,5-dihydro- $1 H$-pyrazoles 1a-c reacted with a variety of primary and secondary amines in molar ratio 1:1 - 1:2 in methanol or THF for 6-24 h yielding 5alkylamino(or dialkylamino)-3-(2-arylvinyl)-5-phenyl-1-tosyl-4,5-dihydro-1H-pyrazoles $2 d-m$ in $36-75 \%$ (Scheme 1, Table 1).

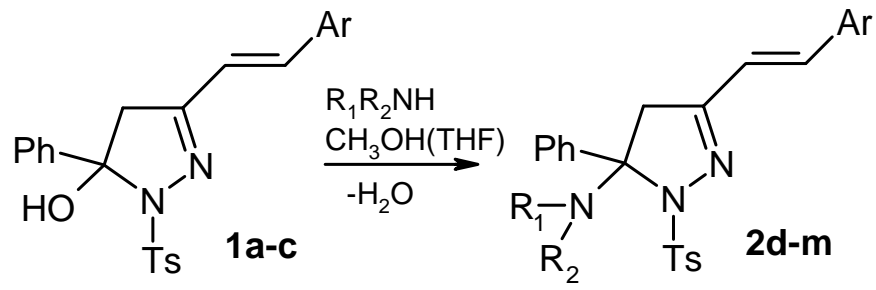

\section{Scheme 1}

Table 1. Synthesis of 5-R-amino-3-(2-arylvinyl)-5-phenyl-1-tosyl-4,5-dihydro-1H-pyrazoles 2d-m

\begin{tabular}{cccccc}
\hline Entry & $\mathrm{R}_{1}$ & $\mathrm{R}_{2}$ & $\mathrm{Ar}$ & Solvent & Compound 2(Yield) \\
\hline 1 & $\mathrm{Me}$ & $\mathrm{H}$ & $\mathrm{Ph}$ & $\mathrm{MeOH}$ & $\mathbf{2 d}(70 \%)$ \\
2 & $\mathrm{Pr}$ & $\mathrm{H}$ & $\mathrm{Ph}$ & $\mathrm{THF}$ & $\mathbf{2 e}(65 \%)$ \\
3 & $\mathrm{Ch}$ & $\mathrm{H}$ & $\mathrm{Ph}$ & $\mathrm{MeOH}$ & $\mathbf{2 f}(69 \%)$ \\
4 & \multicolumn{2}{c}{$\mathrm{CH}_{2} \mathrm{OCH}_{2}$} & $\mathrm{Ph}$ & $\mathrm{THF}$ & $\mathbf{2 g}(50 \%)$ \\
5 & $\mathrm{Pr}$ & $\mathrm{H}$ & $3-\mathrm{Cl}-\mathrm{C}_{6} \mathrm{H}_{4}$ & $\mathrm{MeOH}$ & $\mathbf{2 h}(62 \%)$ \\
6 & $\mathrm{Me}$ & $\mathrm{Me}$ & $3-\mathrm{Cl}_{-}-\mathrm{C}_{6} \mathrm{H}_{4}$ & $\mathrm{MeOH}$ & $\mathbf{2 i}(64 \%)$ \\
7 & $\mathrm{Me}$ & $\mathrm{H}$ & $4-\mathrm{NO}_{2}-\mathrm{C}_{6} \mathrm{H}_{4}$ & $\mathrm{MeOH}$ & $\mathbf{2 j}(75 \%)$ \\
8 & $\mathrm{Ch}$ & $\mathrm{H}$ & $4-\mathrm{NO}_{2}-\mathrm{C}_{6} \mathrm{H}_{4}$ & $\mathrm{MeOH}$ & $\mathbf{2 k}(71 \%)$ \\
9 & $\mathrm{PhCH}$ & $\mathrm{H}$ & $4-\mathrm{NO}_{2}-\mathrm{C}_{6} \mathrm{H}_{4}$ & $\mathrm{MeOH}$ & $\mathbf{2 l}(36 \%)$ \\
10 & $\mathrm{HO}\left(\mathrm{CH}_{2}\right)_{3}$ & $\mathrm{H}$ & $4-\mathrm{NO}_{2}-\mathrm{C}_{6} \mathrm{H}_{4}$ & $\mathrm{THF}$ & $\mathbf{2 m}(46 \%)$ \\
\hline
\end{tabular}

The structures of products $\mathbf{2 d - m}$ were confirmed by ${ }^{1} \mathrm{H}-\mathrm{NMR}$ and IR spectral data (the presence of $\mathrm{N}$-alkyl proton signals and the absorption at $3375-3385 \mathrm{~cm}^{-1}$, corresponding to NH-bond vibrations, respectively ${ }^{11}$ ). The absorption band of the hydroxyl group in starting materials, 5-hydroxy-2-pyrazolines at $3505 \mathrm{~cm}^{-1}$ are not present in the products, 5-amino-2-pyrazolines.

The formation of 5-amino-5-phenyl-1-tosyl-2-pyrazolines $\mathbf{2 d - m}$ proceeds via acyclic intermediate $\mathbf{A}$, which further transforms to the imino-hydrazone intermediate $\mathbf{B}$ and enamino- 
hydrazone B' as a result of the intermolecular nucleophilic addition of the amine to the carbonyl group (Scheme 2). Finally, upon preferential 5-exo-trig-attack of the imines group the intermediates $\mathbf{B}$ or B' undergo cyclization to the 5-amino-2-pyrazolines $\mathbf{2 d - m}$, according to the Baldwin's rule. ${ }^{12}$

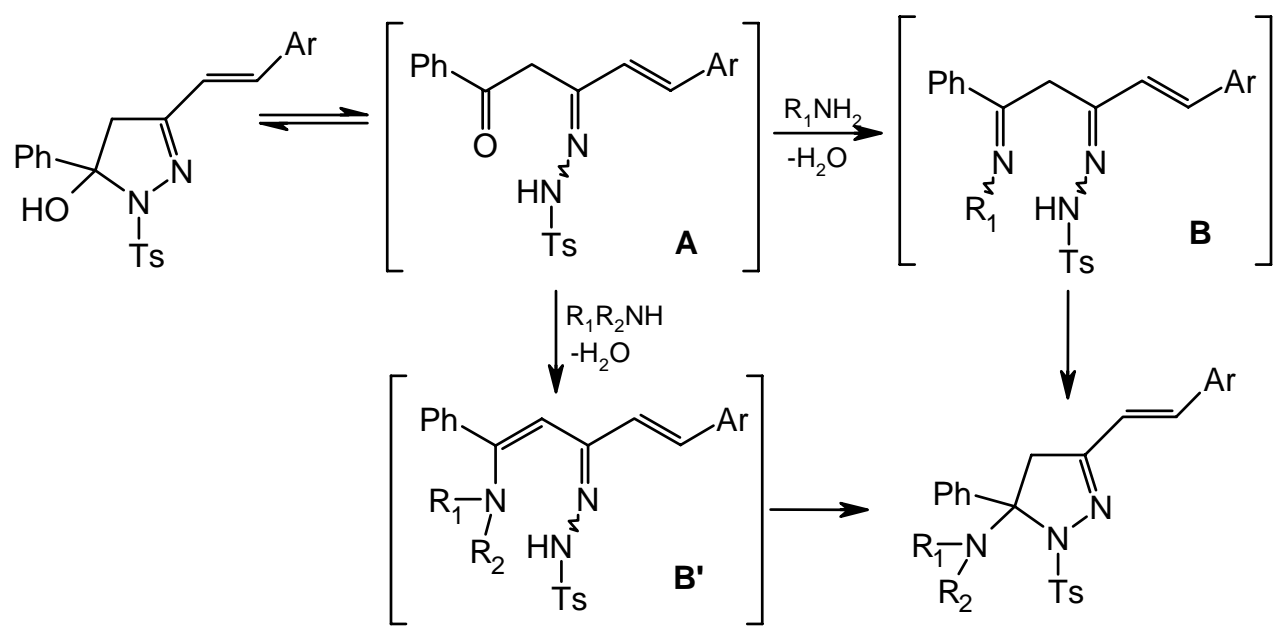

\section{Scheme 2}

Interestingly, substitution of the hydroxyl group by the amine in a case of 5-hydroxy-1-tosyl-2pyrazolines 1a-c proceeds in much milder conditions than in the case of 1-acyl-5-hydroxy-2pyrazolines, ${ }^{13,14}$ which react only in solid phase synthesis conditions, usually at high temperature.

When hydrazine hydrate is used as a nucleophile in the reaction with 5-hydroxy-2-pyrazolines 1a,c,n, the 3(5)-(2-arylvinyl)-5(3)-phenyl-1H-pyrazoles 3a,c,n are obtained with 84-89\% (Scheme 3, Table 2). Compounds 3a,c,n are positional isomers of the pyrazoles that we synthesized earlier in our laboratory. ${ }^{15}$ They are the products of the reaction of hydrazine hydrate with cynnamoyloxiranes and the following dehydration. Asymmetric $\beta, \beta$ '-diaryl-dioxiranylketones upon reaction with hydrazine give $\beta$-hydroxyalkylpyrazoles. Their subsequent dehydration yield a mixture of isomeric 3(5)-aryl-5(3)-(2-phenylvinyl)-1H-pyrazoles and 5(3)-(2-arylvinyl)-3(5)phenyl-1H-pyrazoles. The reaction of 5-hydroxy-2-pyrazolines 1a,c with phenylhydrazine instead of hydrazine hydrate displays deeply colored reaction mixture from which it was not possible to isolate individual compounds.

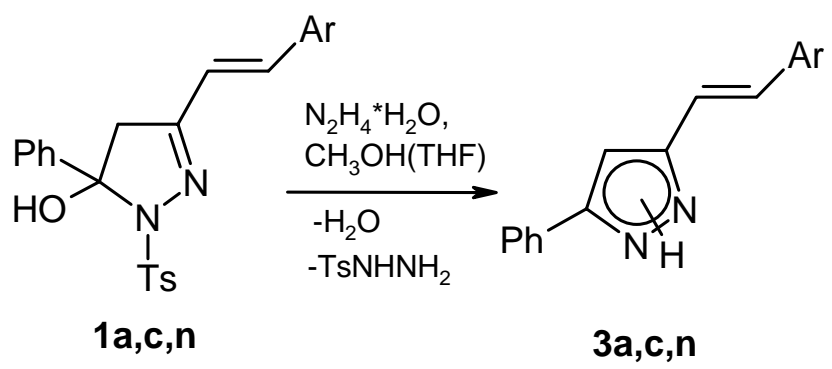

\section{Scheme 3}


Table 2. Synthesis of 3(5)-(2-arylvinyl)-5(3)-phenyl-1H-pyrazoles 3a,c,n

\begin{tabular}{ccc}
\hline Entry & $\mathrm{Ar}$ & Compound 3 (Yield) \\
\hline 1 & $\mathrm{Ph}$ & $\mathbf{3 a}(84 \%)$ \\
2 & $4-\mathrm{NO}_{2}-\mathrm{C}_{6} \mathrm{H}_{4}$ & $\mathbf{3 c}(89 \%)$ \\
3 & $4-\mathrm{Br}^{-} \mathrm{C}_{6} \mathrm{H}_{4}$ & $\mathbf{3 n}(88 \%)$ \\
\hline
\end{tabular}

The IR spectra confirms the structure of pyrazoles 3a,c,n by the presence the N-H vibrations bands at $3264-3232 \mathrm{~cm}^{-1}$. In the ${ }^{1} \mathrm{H}$ NMR spectra there is a singlet at $6.75-6.81 \mathrm{ppm}$ of the hydrogen atom at the 4-position of the azole ring, ${ }^{16}$ the signals of tosyl moiety protons are absent.

The reaction mechanism of 3-(2-arylvinyl)-5-hydroxy-5-phenyl-1-tosyl-2-pyrazolines 1a,c,n with hydrazine includes the nucleophilic attack of the substrate acyclic form, however, diketone bishydrazone $\mathbf{D}$ cyclization occurs with participation of the more nucleophilic $\mathrm{NH}_{2}$ group, rather than the NH group of the hydrazone moieties, thus leading to the 5-tosylhydrazine-2-pyrazoline $\mathbf{E}$ intermediate (Scheme 4). The following elimination of tosylhydrazine affords the final products, 3(5)-(2-arylvinyl)-5(3)-phenyl-1H-pyrazoles 3a,c,n. We were not able to isolate or detect the pyrazolines $\mathbf{E}$ intermediate by the ${ }^{1} \mathrm{H}$ NMR method. It is important to note that the color of the solution of 5-hydroxy-2-pyrazolines become yellow or brightly orange immediately after addition even a drop of hydrazine then the reaction mixture gradually becomes colorless. The observed discoloration indicates breaking the conjugation in the intermediate bis-hydrazones which is present in the tautomeric equilibrium with enhydrazine form.

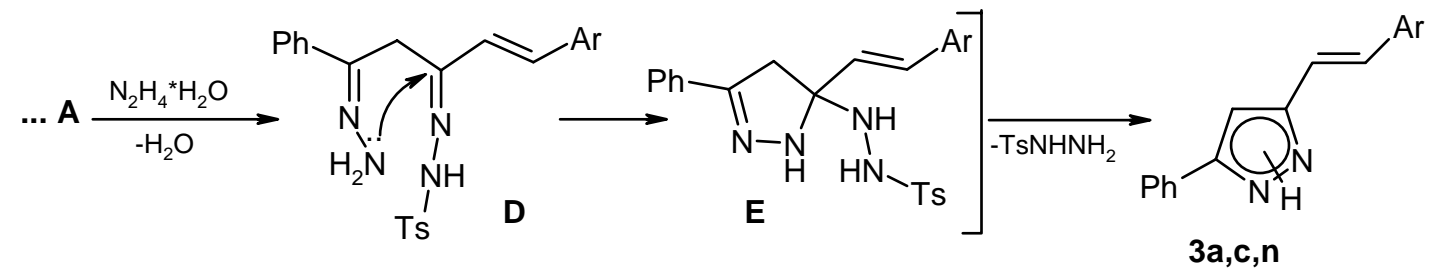

\section{Scheme 4}

We conclude, that the 5-hydroxy-5-phenyl-1-tosyl-2-pyrazolines can be employed as convenient substrates in the reactions with amines and hydrazine for the synthesis of 5-amine-3-(2-arylvinyl)-5phenyl-1-tosyl-4,5-dihidro-1H-pyrazoles and 3(5)-(2-arylvinyl)-5(3)-phenyl-1H-pyrazoles.

\section{Experimental Section}

General Procedures. Synthesis of 3-(2-arylvinyl)-5-methyl- or 5-dimethylamino-1-tosyl-5phenyl-4,5-dihydro-1H-pyrazoles (2d,i,j). Double molar excess of methylamine or dimethylamine in methanol was added to the colorless solution of 5-hydroxy-2-pyrazoline 1a-c in $15 \mathrm{ml}$ of methanol. After the addition of the first drop of the amine the reaction mixture color 
instantaneously becomes orange. The mixture was kept at r.t. for 16-24 hours. Then the solvent and an excess of the amine were removed in vacuum and the residue was crystallized from the mixture of chloroform-methanol (1:10). Precipitated solids of 5-aminopyrazolines $\mathbf{2 d}, \mathbf{i}, \mathbf{j}$ were separated by filtration.

5-Methylamino-5-phenyl-3-(2-phenylvinyl)-1-tosyl-4,5-dihydro-1 $\mathrm{H}$-pyrazole (2d). Yield $70 \%$. Mp. ${ }^{174-175^{\circ} \mathrm{C} .}{ }^{1} \mathrm{H}$ NMR (400 MHz, $\left.\mathrm{CDCl}_{3}\right): \delta 2.41\left(3 \mathrm{H}, \mathrm{s}, \mathrm{C}_{6} \mathrm{H}_{4}-\mathrm{CH}_{3}\right), 2.46\left(3 \mathrm{H}, \mathrm{s}, \mathrm{CH}_{3} \mathrm{NH}\right)$, $3.16\left(1 \mathrm{H}, \mathrm{d}, J=18.0, \mathrm{CH}_{2}\right), 3.40\left(1 \mathrm{H}, \mathrm{d}, J=18.0, \mathrm{CH}_{2}\right), 6.63(1 \mathrm{H}, \mathrm{d}, J=16.4, \mathrm{CH}=), 7.07(1 \mathrm{H}, \mathrm{d}, J=$ 16.4, $\mathrm{CH}=), 7.19-7.50(12 \mathrm{H}, \mathrm{m}$, arom $), 7.65\left(2 \mathrm{H}, \mathrm{d}, J=8.3, \mathrm{C}_{6} \mathbf{H}_{4}-\mathrm{CH}_{3}\right)$. Anal. Calcd for $\mathrm{C}_{25} \mathrm{H}_{25} \mathrm{~N}_{3} \mathrm{O}_{2} \mathrm{~S}: \mathrm{C} 69.58, \mathrm{H}$ 5.84, N 9.74. Found: C 69.44, H 5.98, N 9.87.

3-[2-(3-Chlorophenyl)vinyl]-5-dimethylamino-5-phenyl-1-tosyl-4,5-dihydro-1 $\mathrm{H}$-pyrazole (2i). Yield 64\%. Mp. $154-156^{\circ} \mathrm{C} .{ }^{1} \mathrm{H}$ NMR $\left(400 \mathrm{MHz}, \mathrm{CDCl}_{3}\right): \delta 2.30\left(6 \mathrm{H}, \mathrm{s},\left(\mathrm{CH}_{3}\right)_{2} \mathrm{~N}\right), 2.40(3 \mathrm{H}, \mathrm{s}$, $\left.\mathrm{C}_{6} \mathrm{H}_{4}-\mathrm{CH}_{3}\right), 3.26\left(1 \mathrm{H}, \mathrm{d}, J=18.0, \mathrm{CH}_{2}\right), 3.47\left(1 \mathrm{H}, \mathrm{d}, J=18.0, \mathrm{CH}_{2}\right), 6.60(1 \mathrm{H}, \mathrm{d}, J=16.6, \mathrm{CH}=), 7.06$ $(1 \mathrm{H}, \mathrm{d}, J=16.6, \mathrm{CH}=), 7.21-7.51\left(11 \mathrm{H}, \mathrm{m}\right.$, arom), $7.64\left(2 \mathrm{H}, \mathrm{d}, J=8.3, \mathrm{C}_{6} \mathbf{H}_{4}-\mathrm{CH}_{3}\right)$. Anal. Calcd for $\mathrm{C}_{26} \mathrm{H}_{26} \mathrm{ClN}_{3} \mathrm{O}_{2} \mathrm{~S}$ : C 65.06, H 5.46, N 8.75. Found: C 64.87, H 5.54, N 8.89.

5-Methylamino-3-[2-(4-nitrophenyl)vinyl]-5-phenyl-1-tosyl-4,5-dihydro-1 $\mathrm{H}$-pyrazole (2j).

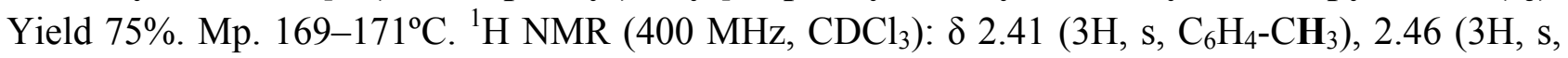
$\left.\mathrm{CH}_{3} \mathrm{NH}\right), 3.17\left(1 \mathrm{H}, \mathrm{d}, J=18.2, \mathrm{CH}_{2}\right), 3.42\left(1 \mathrm{H}, \mathrm{d}, J=18.2, \mathrm{CH}_{2}\right), 6.65(1 \mathrm{H}, \mathrm{d}, J=16.6, \mathrm{CH}=)$, $7.19(1 \mathrm{H}, \mathrm{d}, J=16.6, \mathbf{C H}=), 7.21-7.35(7 \mathrm{H}, \mathrm{m}, \operatorname{arom}), 7.53\left(2 \mathrm{H}, \mathrm{d}, J=9.0, \mathrm{C}_{6} \mathbf{H}_{4}-\mathrm{NO}_{2}\right), 7.57(2 \mathrm{H}$, d, $\left.J=8.4, \mathrm{C}_{6} \mathrm{H}_{4}-\mathrm{CH}_{3}\right), 8.20\left(2 \mathrm{H}, \mathrm{d}, J=9.0, \mathrm{C}_{6} \mathbf{H}_{4}-\mathrm{NO}_{2}\right)$. Anal. Calcd for $\mathrm{C}_{25} \mathrm{H}_{24} \mathrm{~N}_{4} \mathrm{O}_{4} \mathrm{~S}: \mathrm{C} 63.01, \mathrm{H}$ 5.08, N 11.76. Found: C 62.84, H 5.31, N 11.82.

General procedure. Synthesis of 3-(2-arylvinyl)-5-R-amino-1-tosyl-5-phenyl-4,5-dihydro- $1 H$ pyrazoles (2e,f,g,h,k,l,m)

Procedure has been described elsewhere. ${ }^{8}$

The spectral data of 3-(2-phenylvinyl)-5-cyclohexylamine-1-tosyl-5-phenyl-4,5-dihydro- $1 \mathrm{H}$ pyrazole $\mathbf{2 f}$ have been reported elsewhere. ${ }^{8}$

5-Phenyl-3-(2-phenylvinyl)-5-propylamino-1-tosyl-4,5-dihydro-1 $H$-pyrazole (2e). Yield $65 \%$. Light yellow oil. ${ }^{1} \mathrm{H}$ NMR $\left(400 \mathrm{MHz}, \mathrm{CDCl}_{3}\right): \delta 0.96\left(3 \mathrm{H}, \mathrm{T}, J=7.3, \mathrm{CH}_{3} \mathrm{CH}_{2} \mathrm{CH}_{2}\right), 1.60(2 \mathrm{H}$, $\left.\mathrm{m}, \mathrm{CH}_{3} \mathrm{CH}_{2} \mathrm{CH}_{2}\right), 2.40\left(3 \mathrm{H}, \mathrm{s}, \mathrm{C}_{6} \mathrm{H}_{4}-\mathrm{CH}_{3}\right), 2.57\left(2 \mathrm{H}, \mathrm{m}, \mathrm{CH}_{3} \mathrm{CH}_{2} \mathrm{CH}_{2}\right), 3.14$ (1H, d, $J=18.0, \mathrm{CH}_{2}$ ), $3.37\left(1 \mathrm{H}, \mathrm{d}, J=18.0, \mathrm{CH}_{2}\right), 6.61(1 \mathrm{H}, \mathrm{d}, J=16.4, \mathrm{CH}=), 7.05$ (1H, d, $\left.J=16.4, \mathrm{CH}=\right), 7.19-7.43$ $(12 \mathrm{H}, \mathrm{m}, \operatorname{arom}), 7.59\left(2 \mathrm{H}, \mathrm{d}, J=8.3, \mathrm{C}_{6} \mathbf{H}_{4}-\mathrm{CH}_{3}\right)$. IR $\left(\mathrm{KBr}, \mathrm{cm}^{-1}\right): v=3378(\mathrm{NH}), 2936(\mathrm{CH}), 1368$ $(\mathrm{S}=\mathrm{O}), 1168(\mathrm{~S}=\mathrm{O})$ Anal. Calcd for $\mathrm{C}_{27} \mathrm{H}_{29} \mathrm{~N}_{3} \mathrm{O}_{2} \mathrm{~S}$ : C 70.56, H 6.36, N 9.14. Found: C 70.40, H $6.53, \mathrm{~N} 9.23$.

5-Morpholino-5-phenyl-3-(2-phenylvinyl)-1-tosyl-4,5-dihydro-1H-pyrazole (2g). Yield $50 \%$. Light yellow oil. ${ }^{1} \mathrm{H}$ NMR $\left(400 \mathrm{MHz}, \mathrm{CDCl}_{3}\right): \delta 2.40\left(3 \mathrm{H}, \mathrm{s}, \mathrm{C}_{6} \mathrm{H}_{4}-\mathrm{CH}_{3}\right), 2.99(4 \mathrm{H}, \mathrm{m}$, $\left.\mathrm{CH}_{2} \mathrm{NCH}_{2}\right), 3.46\left(1 \mathrm{H}, \mathrm{d}, J=17.9, \mathrm{CH}_{2}\right), 3.64\left(1 \mathrm{H}, \mathrm{d}, J=17.9, \mathrm{CH}_{2}\right), 3.76\left(4 \mathrm{H}, \mathrm{m}, \mathrm{CH}_{2} \mathrm{OCH}_{2}\right), 6.63$ $(1 \mathrm{H}, \mathrm{d}, J=16.4, \mathrm{CH}=), 7.02(1 \mathrm{H}, \mathrm{d}, J=16.4, \mathrm{CH}=), 7.22-7.71(14 \mathrm{H}, \mathrm{m}$, arom). Anal. Calcd for $\mathrm{C}_{28} \mathrm{H}_{29} \mathrm{~N}_{3} \mathrm{O}_{3} \mathrm{~S}: \mathrm{C} 68.97, \mathrm{H}$ 5.99, N 8.62. Found: C 68.81, H 6.14, N 8.78.

3-(2-(3-Chlorophenyl)vinyl)-5-phenyl-5-propylamino-1-tosyl-4,5-dihydro-1 $H$-pyrazole (2h). Yield 62\%. Light yellow oil. ${ }^{1} \mathrm{H}$ NMR $\left(400 \mathrm{MHz}, \mathrm{CDCl}_{3}\right): \delta 0.97(3 \mathrm{H}, \mathrm{T}, J=7.3$, 
$\left.\mathrm{CH}_{3} \mathrm{CH}_{2} \mathrm{CH}_{2}\right), 1.61\left(2 \mathrm{H}, \mathrm{m}, \mathrm{CH}_{3} \mathrm{CH}_{2} \mathrm{CH}_{2}\right), 2.41\left(3 \mathrm{H}, \mathrm{s}, \mathrm{C}_{6} \mathrm{H}_{4}-\mathrm{CH}_{3}\right), 2.56\left(2 \mathrm{H}, \mathrm{m}, \mathrm{CH}_{3} \mathrm{CH}_{2} \mathrm{CH}_{2}\right)$, $3.12\left(1 \mathrm{H}, \mathrm{d}, J=18.0, \mathrm{CH}_{2}\right), 3.35\left(1 \mathrm{H}, \mathrm{d}, J=18.0, \mathrm{CH}_{2}\right), 6.53(1 \mathrm{H}, \mathrm{d}, J=16.4, \mathrm{CH}=), 7.04(1 \mathrm{H}, \mathrm{d}, J$ $=16.4, \mathbf{C H}=), 7.21-7.51(11 \mathrm{H}, \mathrm{m}$, arom $), 7.59\left(2 \mathrm{H}, \mathrm{d}, J=8.3, \mathrm{C}_{6} \mathbf{H}_{4}-\mathrm{CH}_{3}\right) . \mathrm{IR}\left(\mathrm{KBr}, \mathrm{cm}^{-1}\right): v=3375$ $(\mathrm{NH}), 2935(\mathrm{CH}), 1359(\mathrm{~S}=\mathrm{O}), 1168(\mathrm{~S}=\mathrm{O})$. Anal. Calcd for $\mathrm{C}_{27} \mathrm{H}_{28} \mathrm{ClN}_{3} \mathrm{O}_{2} \mathrm{~S}: \mathrm{C}$ 65.64, H 5.71, N 8.51. Found: C 65.49, H 5.87, N 8.34.

\section{5-Cyclohexylamino-3-[2-(4-nitrophenyl)vinyl]-5-phenyl-1-tosyl-4,5-dihydro-1 $H$-pyrazole} (2k). Yield 71\%. Light yellow oil. ${ }^{1} \mathrm{H}$ NMR $\left(400 \mathrm{MHz}, \mathrm{CDCl}_{3}\right)$ : $\delta 1.21-2.27\left(10 \mathrm{H}, \mathrm{m}, \mathrm{C}_{6} \mathbf{H}_{11}\right), 2.39$ $\left(3 \mathrm{H}, \mathrm{s}, \mathrm{C}_{6} \mathrm{H}_{4}-\mathrm{CH}_{3}\right), 2.83\left(1 \mathrm{H}, \mathrm{m}, \mathrm{C}_{6} \mathbf{H}_{11}\right), 3.17\left(1 \mathrm{H}, \mathrm{d}, J=18.0, \mathrm{CH}_{2}\right), 3.55\left(1 \mathrm{H}, \mathrm{d}, J=18.0, \mathrm{CH}_{2}\right), 6.65$ $(1 \mathrm{H}, \mathrm{d}, J=16.4, \mathbf{C H}=), 7.18(1 \mathrm{H}, \mathrm{d}, J=16.4, \mathrm{CH}=), 7.21-7.36(7 \mathrm{H}, \mathrm{m}, \operatorname{arom}), 7.53\left(2 \mathrm{H}, \mathrm{d}, J=9.0, \mathrm{C}_{6} \mathbf{H}_{4}-\right.$ $\left.\mathrm{NO}_{2}\right), 7.57\left(2 \mathrm{H}, \mathrm{d}, J=8.4, \mathrm{C}_{6} \mathbf{H}_{4}-\mathrm{CH}_{3}\right), 8.20\left(2 \mathrm{H}, \mathrm{d}, J=9.0, \mathrm{C}_{6} \mathrm{H}_{4}-\mathrm{NO}_{2}\right)$. Anal. Calcd for $\mathrm{C}_{30} \mathrm{H}_{32} \mathrm{~N}_{4} \mathrm{O}_{4} \mathrm{~S}$ : C 66.16, H 5.92, N 10.29. Found: C 66.03, H 6.00, N 10.45.

5-Benzylamino-3-[(2-(4-nitrophenyl)vinyl]-5-phenyl-1-tosyl-4,5-dihydro-1 H-pyrazole (2l). Yield 36\%. Mp. $230^{\circ} \mathrm{C}$ (decomp.). ${ }^{1} \mathrm{H}$ NMR $\left(400 \mathrm{MHz}, \mathrm{CDCl}_{3}\right): \delta 2.42\left(3 \mathrm{H}, \mathrm{s}, \mathrm{C}_{6} \mathrm{H}_{4}-\mathrm{CH}_{3}\right), 3.18(1 \mathrm{H}$, d, $\left.J=18.0, \mathrm{CH}_{2}\right), 3.44\left(1 \mathrm{H}, \mathrm{d}, J=18.0, \mathrm{CH}_{2}\right), 4.82\left(2 \mathrm{H}, \mathrm{d}, J=1.1, \mathrm{CH}_{2} \mathrm{Ph}\right), 6.57(1 \mathrm{H}, \mathrm{d}, J=16.4$, $\mathrm{CH}=), 7.11-7.80(17 \mathrm{H}, \mathrm{m}$, arom, $\mathrm{CH}=), 8.19\left(2 \mathrm{H}, \mathrm{d}, J=8.7, \mathrm{C}_{6} \mathbf{H}_{4}-\mathrm{NO}_{2}\right), 8.39$ (1H, br.t, NH). Anal. Calcd for $\mathrm{C}_{31} \mathrm{H}_{28} \mathrm{~N}_{4} \mathrm{O}_{4} \mathrm{~S}$ : C 67.37, H 5.11, N 10.14. Found: C 67.20, H 5.34, N 10.25.

5-(3-Hydroxypropyl)amino-3-[2-(4-nitrophenyl)vinyl]-5-phenyl-1-tosyl-4,5-dihydro-1 Hpyrazole $(2 \mathrm{~m})$. Yield $46 \%$. Light yellow oil. ${ }^{1} \mathrm{H}$ NMR $\left(400 \mathrm{MHz}, \mathrm{CDCl}_{3}\right): \delta 1.88(2 \mathrm{H}, \mathrm{m}$, $\left.\mathrm{HOCH}_{2} \mathrm{CH}_{2} \mathrm{CH}_{2} \mathrm{NH}\right), 2.41\left(3 \mathrm{H}, \mathrm{s}, \mathrm{C}_{6} \mathrm{H}_{4}-\mathrm{CH}_{3}\right), 2.69\left(2 \mathrm{H}, \mathrm{m}, \mathrm{HOCH}_{2} \mathrm{CH}_{2} \mathrm{CH}_{2} \mathrm{NH}\right), 3.18(1 \mathrm{H}, \mathrm{d}, J=$ 18.0, $\left.\mathrm{CH}_{2}\right), 3.42\left(1 \mathrm{H}, \mathrm{d}, J=18.0, \mathrm{CH}_{2}\right), 3.86\left(2 \mathrm{H}, \mathrm{m}, \mathrm{HOCH}_{2} \mathrm{CH}_{2} \mathrm{CH}_{2} \mathrm{NH}\right), 6.45(1 \mathrm{H}, \mathrm{d}, J=16.4$, $\mathrm{CH}=), 7.18(1 \mathrm{H}, \mathrm{d}, J=16.4, \mathrm{CH}=), 7.20-7.56(11 \mathrm{H}, \mathrm{m}, \operatorname{arom}), 8.20\left(2 \mathrm{H}, \mathrm{d}, J=8.7, \mathrm{C}_{6} \mathbf{H}_{4}-\mathrm{NO}_{2}\right)$. Anal. Calcd for $\mathrm{C}_{27} \mathrm{H}_{28} \mathrm{~N}_{4} \mathrm{O}_{5} \mathrm{~S}$ : C 62.29, H 5.42, N 10.76. Found: C 62.02, H 5.57, N 10.59.

General procedure. Synthesis of 3(5)-(2-arylvinyl)-5(3)-phenyl-1H-pyrazoles $(3 \mathrm{a}, \mathbf{c}, \mathbf{n})$. To the solution of $2.5 \mathrm{mmol} 5$-hydroxy-2-pyrazolines 1a,c,n in $20 \mathrm{ml} \mathrm{MeOH-THF} \mathrm{mixture} \mathrm{(5:1)} 0.2 \mathrm{ml}$ hydrazine hydrate was added. Reaction mixture was left at $25^{\circ} \mathrm{C}$ for 12 hours. Solvent was removed in vacuum and residue was diluted with ether. Solid pyrazoles 3a,c,n were filtered off as a yellow crystals.

5(3)-Phenyl-3(5)-(2-phenylvinyl)-1H-pyrazole (3a). Yield 84\%. Mp. 136-138 ${ }^{\circ} \mathrm{C} .{ }^{1} \mathrm{H}$ NMR (400 $\left.\mathrm{MHz}, \mathrm{CDCl}_{3}\right): \delta 6.75(1 \mathrm{H}, \mathrm{s}, \mathrm{C}(4)-\mathbf{H}), 7.04(1 \mathrm{H}, \mathrm{d}, J=16.4, \mathrm{CH}=), 7.11(1 \mathrm{H}, \mathrm{d}, J=16.4, \mathrm{CH}=)$, 7.17-7.73 (10H, m, arom). IR $\left(\mathrm{KBr}, \mathrm{cm}^{-1}\right): v=3264(\mathrm{NH}), 1596(\operatorname{arom}), 957(\mathrm{HC}=)$ Anal. Calcd for $\mathrm{C}_{17} \mathrm{H}_{14} \mathrm{~N}_{2}$ : C 82.90, H 5.73, N 11.37. Found: C 82.85, H 5.84, N 11.45.

3(5)-[2-(4-Nitrophenyl)vinyl]-5(3)-phenyl-1 $\boldsymbol{H}$-pyrazole (3c). Yield $89 \%$. Mp. $187-188^{\circ} \mathrm{C} .{ }^{1} \mathrm{H}$ NMR $\left(400 \mathrm{MHz}, \mathrm{CDCl}_{3}\right): \delta 6.81(1 \mathrm{H}, \mathrm{s}, \mathrm{C}(4)-\mathbf{H}), 7.17(1 \mathrm{H}, \mathrm{d}, J=16.6, \mathrm{CH}=), 7.24(1 \mathrm{H}, \mathrm{d}$, $J=16.6, \mathbf{C H}=), 7.35-7.47\left(3 \mathrm{H}, \mathrm{m}, \mathrm{C}_{6} \mathbf{H}_{5}\right), 7.60\left(2 \mathrm{H}, \mathrm{d}, J=8.7, \mathrm{C}_{6} \mathbf{H}_{4}\right), 7.67\left(2 \mathrm{H}, \mathrm{m}, \mathrm{C}_{6} \mathbf{H}_{5}\right), 8.21$ $\left(2 \mathrm{H}, \mathrm{d}, J=8.7, \mathrm{C}_{6} \mathbf{H}_{4}\right) . \mathrm{IR}\left(\mathrm{KBr}, \mathrm{cm}^{-1}\right): v=3232(\mathrm{NH}), 1593(\operatorname{arom}), 1508\left(\mathrm{NO}_{2}\right), 1344\left(\mathrm{NO}_{2}\right)$, $960(\mathrm{HC}=)$. Anal. Calcd for $\mathrm{C}_{17} \mathrm{H}_{13} \mathrm{~N}_{3} \mathrm{O}_{2}$ : C 70.09, H 4.50, N 14.42. Found: C 69.91, H 4.67, N 14.38.

3(5)-[2-(4-Bromophenyl)vinyl]-5(3)-phenyl-1H-pyrazole (3n). Yield 88\%. Mp. 223-225 ${ }^{\circ} \mathrm{C}$. ${ }^{1} \mathrm{H}$ NMR $\left(400 \mathrm{MHz}, \mathrm{CDCl}_{3}\right): \delta 6.75(1 \mathrm{H}, \mathrm{s}, \mathrm{C}(4)-\mathbf{H}), 7.04(2 \mathrm{H}, \mathrm{s}, \mathrm{CH}=\mathrm{CH}), 7.32-7.48(7 \mathrm{H}, \mathrm{m}$, 
arom), 7.69 (2H, m, arom). IR $\left(\mathrm{KBr}, \mathrm{cm}^{-1}\right): v=3263(\mathrm{NH}), 1595$ (arom), $958(\mathrm{HC}=)$. Anal. Calcd for $\mathrm{C}_{17} \mathrm{H}_{13} \mathrm{BrN}_{2}$ : C 62.79, H 4.03, N 8.61. Found: C 62.62, H 4.26, N 8.79.

\section{Acknowledgements}

We thank Prof. Oleg Kulinkovich for the assistance in carrying out of spectroscopic analysis.

\section{References}

1. Valter, R. E. Ring-chain isomerisation in organic chemistry (In Russian); Zinatne: Riga, 1978.

2. Zelenin, K. N.; Alekseyev, V. V.; Pihlaja, K.; Ovcharenko, V. V. Russ. Chem. Bull. (Engl. Transl.) 2002, 51, 205.

3. Zelenin, K. N.; Alekseyev, V. V. Chem. Heterocycl. Compd. (Engl. Transl.) 1992, 28, 708.

4. Erschov, A. Yu.; Gindin, V. A.; Gribanov, A. V. Zh. Org. Khim. (Russ. J. Org. Chem.) 1997, $33,438$.

5. Yakimovitch, S. I.; Zerova, I. V.; Zelenin, K. N.; Alekseyev, V. V.; Tugusheva, A. R. Zh. Org. Khim. (Russ. J. Org. Chem.) 1997, 33, 418.

6. Yakimovitch, S. I.; Zerova, I. V. Zh. Org. Khim. (Russ. J. Org. Chem.) 1993, 29, 905.

7. Kozminykh, V. O.; Goncharov, V. I.; Kozminykh, E. N.; Oborin, D. B. Chem. Heterocycl. Compd. (Ingl. Transl.) 2006, 42, 698.

8. Kuz'menok, N. M.; Koval'chuk, T. A.; Zvonok, A. M. Synlett 2005, 485.

9. Zelenin, K. N.; Alekseyev, V. V.; Tygysheva, A. R. Tetrahedron 1995, 51, 11251.

10. Zelenin, K. N.; Tugusheva, A. R.; Yakimovich, S. I.; Alekseev, V. V.; Zerova E. V. Chem. Heterocycl. Compd. (Ingl. Transl.) 2002, 38, 668.

11. Nakanishi, K.; Solomon, P. H. Infrared Absorption Spec-troscopy, 2nd ed., Emerson Adams Pr. Inc., 1998.

12. Baldwin, J. E. Chem. Comm. 1976, 734.

13. Ershov, A. Yu. Zh. Org. Khim. (Russ. J. Org. Chem.) 1995, 31, 1057.

14. Ershov, A. Yu.; Koshmina, N. V. Zh. Org. Khim. (Russ. J. Org. Chem.) 1998, 34, 953.

15. Zvonok, A. M.; Kuz'menok, N. M.; Stanishevsky, L. S. Khim. Geterotsikl. Soedin. 1990, $5,633$.

16. Batterham, T.J. NMR spectra of simple heterocycles. New York. 1973. 